\title{
Suporte social e assistencial de pessoas com demência e seus cuidadores através de um aplicativo móvel de saúde
}

\author{
Social and care support for people with dementia and their caregivers through a mobile health \\ application
}

Apoyo social y asistencial a persona con demencia y sus cuidadores a través de una aplicación para dispositivos móviles

\section{Resumo}

Objetivo da pesquisa foi analisar a utilização do Sistema Móvel de Assistência ao Idoso (SMAI) no cuidado de pessoas com demência por meio das mensagens trocadas entre os familiares cuidadores e os profissionais de saúde. Trata-se de estudo de abordagem qualitativa, baseado no referencial fenomenológico descritivo, sobre a experiência de utilização do aplicativo SMAI na produção do cuidado em um serviço de geriatria e gerontologia. Os dados foram analisados com auxílio do software IRAMUTEQ e a técnica da Classificação Hierárquica Descendente (CHD) para estimar a frequência das palavras por meio da classificação dos segmentos de textos em função dos vocabulários e análise lexical das palavras. Foram realizadas análises estatísticas sobre dois corpus textuais, sendo um deles resultado das mensagens enviadas pelos cuidadores e outro com as respostas dos profissionais de saúde. Trinta e quatro familiares cuidadores de pessoas com demência e cinco profissionais de saúde participaram do estudo. A utilização do sistema traduziu-se na organização das consultas; no manejo das alterações comportamentais; no monitoramento remoto e no gerenciamento do cuidado pela equipe de saúde do serviço, além do estreitamento do vínculo e melhoria da comunicação. As respostas dos profissionais por meio do aplicativo apontaram uma demanda relacionada a organização do cuidado da pessoa com demência no domicílio. O aplicativo SMAI funcionou como uma ferramenta inovadora de suporte social e assistencial aos familiares cuidadores que receberam orientações acerca do cuidar de uma pessoa com demência e suas especificidades. As transformações tecnológicas e sociais podem contribuir para o desenvolvimento de modelos de cuidados à saúde cada vez mais inovadores. O estudo abre perspectivas para o desenvolvimento de aplicativos móveis que promovam o cuidado integral das pessoas com demência e ofereçam uma rede de suporte social aos familiares cuidadores.

Palavras-chave: Cuidadores; Idoso; Comunicação; Aplicativos móveis; Telemedicina. 


\begin{abstract}
The objective of the research was to analyze the use of the Mobile System for Elderly Monitoring (Sistema Móvel de Assistência ao Idoso, SMAI) in the care of people with dementia through the messages exchanged between family caregivers and health professionals. This is a qualitative study, based on a descriptive phenomenological framework, on the experience of using the SMAI application in the production of care in a geriatrics and gerontology service. Data were analyzed using the IRAMUTEQ software and Descending Hierarchical Classification technique to estimate the frequency of words by classifying text segments according to vocabularies and lexical analysis of words. Statistical analyzes were carried out on two textual corpus, one of which was the result of messages sent by caregivers and the other with the answers of health professionals. Thirty-four family caregivers of people with dementia and five health professionals participated in the study. The use of the system resulted in the organization of consultations; in the management of behavioral changes; in remote monitoring and care management by the service's health team, in addition to strengthening the bond and improving communication. The answers of professionals through the application pointed to a demand related to the organization of care for people with dementia at home. The SMAI application worked as an innovative tool for social and assistance support for family caregivers who received guidance on caring for a person with dementia and its specificities. Technological and social transformations can contribute to the development of increasingly innovative health care models. The study opens perspectives for the development of mobile applications that promote comprehensive care for people with dementia and offer a social support network to family caregivers.
\end{abstract}

Keywords: Caregivers; Aged; Communication; Mobile applications; Telemedicine.

\title{
Resumen
}

El objetivo de la investigación fue analizar el uso del Sistema Móvil de Asistencia a Ancianos (Sistema Móvel de Assistência ao Idoso, SMAI) en el cuidado de personas con demencia a través de los mensajes intercambiados entre cuidadores familiares y profesionales de la salud. Este es un estudio cualitativo, basado en un marco fenomenológico descriptivo, sobre la experiencia de uso de la aplicación SMAI en la producción de cuidados en un servicio de geriatría y gerontología. Los datos fueron analizados utilizando el software IRAMUTEQ y la técnica de Clasificación Jerárquica Descendente para estimar la frecuencia de palabras mediante la clasificación de segmentos de texto según vocabularios y análisis léxico de palabras. Se realizaron análisis estadísticos sobre dos corpus textuales, uno de los cuales fue el resultado de los mensajes enviados por los cuidadores y el otro con las respuestas de los profesionales de la salud. Treinta y cuatro cuidadores familiares de personas con demencia y cinco profesionales de la salud participaron en el estudio. El uso del sistema resultó en la organización de consultas; en el manejo de cambios de comportamiento; en el seguimiento remoto y la gestión de la atención por parte del equipo de salud del servicio, además de fortalecer el vínculo y mejorar la comunicación. Las respuestas de los profesionales a través de la aplicación apuntaron a una demanda relacionada con la organización del cuidado de las personas con demencia en el domicilio. La aplicación SMAI funcionó como una herramienta innovadora de apoyo social y asistencial para cuidadores familiares que recibieron orientación sobre el cuidado de una persona con demencia y sus especificidades. Las transformaciones tecnológicas y sociales pueden contribuir al desarrollo de modelos de atención de la salud cada vez más innovadores. El estudio abre perspectivas para el desarrollo de aplicaciones móviles que promuevan la atención integral a las personas con demencia y ofrezcan una red social de apoyo a los cuidadores familiares.

Palabras clave: Cuidadores; Anciano; Comunicación; Aplicaciones móviles; Telemedicina.

\section{Introdução}

A produção do cuidado em saúde se destaca como um conceito que amplia os horizontes de profissionais, serviços e sistemas de saúde, valorizando a dimensão da subjetividade que envolve o acolhimento e a humanização. Representa um lugar de produção de atos, ações, procedimentos e cuidados visando a promoção da saúde (Carnut, 2017).

A pandemia de Covid-19, doença infecciosa causada pelo vírus SARS-Cov-2 ou novo Coronavírus, impactou diretamente a sociedade, o setor saúde e a economia global resultando em mudanças profundas nas práticas e no modelo de atenção à saúde. Os serviços de saúde tiveram que enfrentar essa realidade, buscando novos conceitos e modelos assistenciais que respondessem os problemas de saúde vivenciados pela população. Em diversos países, foi realizado um esforço no sentido de implementar estratégias de telemedicina, como teleconsultas, prescrição online de medicamentos e exames em plataformas digitais, contribuindo para uma disseminação destes serviços (Ohannessian et al., 2020).

Os idosos se configuram como um dos grupos mais vulneráveis ao novo Coronavírus, devido à maior susceptibilidade ao agravamento da Covid-19, o que pode estar associado ao declínio das respostas imunológicas e inflamatórias (Mueller et al., 2020; Cuffaro, 2020). Estima-se haver cerca de 46,8 milhões de pessoas com demência no mundo, sendo que este número 
praticamente irá dobrar a cada 20 anos, chegando a 74,7 milhões em 2030 e a 131,5 milhões em 2050 (Alzheimer's Disease International, 2015). No Brasil, onde a população acima dos 60 anos se aproxima de 29 milhões, estima-se que quase 2 milhões de pessoas têm demência e cerca de 40 a $60 \%$ apresentam doença de Alzheimer, causa mais comum de demência (IBGE, 2018).

Esse fenômeno do envelhecimento tem criado uma demanda crescente por serviços que atendam as necessidades de saúde e sociais destas pessoas, além de políticas públicas que promovam o investimento em tecnologias de cuidado acessíveis, seguras e eficazes. Diante deste cenário, surge o desafio de incentivar e fortalecer as ações de telemedicina e telecuidado voltadas para pessoas com demência e os familiares cuidadores, utilizando estratégias de promoção da saúde e uma rede de suporte social e assistencial (Rathnayake, et al., 2019)

No contexto das tecnologias móveis em saúde, as ações de cuidado são desenvolvidas de maneira remota, envolvendo uma diversidade de dispositivos (sensores, aplicativos para smartphones, tablets e sistemas de informação em saúde), desenvolvidos com o intuito de auxiliar na prestação de cuidados que atendam as necessidades de pessoas, famílias, profissionais de saúde e serviços de saúde (Silva, et al., 2020; Yousaf, et al., 2019). Nesse ambiente digital, os aplicativos de saúde surgem como um recurso potencialmente capaz de transformar o processo de trabalho em saúde e suas relações (Sim, 2019; Rathnayake, et al., 2019).

Este estudo apresenta a experiência de utilização do aplicativo SMAI (Sistema Móvel de Assistência ao Idoso) na produção do cuidado em um serviço de Geriatria e Gerontologia. Tendo como objetivo analisar a utilização do aplicativo SMAI no cuidado de pessoas com demência por meio da troca de mensagens entre os familiares cuidadores e os profissionais de saúde.

\section{Metodologia}

Trata-se de estudo de abordagem qualitativa, baseado no referencial fenomenológico descritivo de Porter (2007), sobre a experiência de utilização do aplicativo SMAI na produção do cuidado em um serviço de geriatria e gerontologia. Os estudos fenomenológicos descritivos se caracterizam pela atribuição de significados ao fenômeno por quem os experimentou (Polit \& Beck, 2011). A análise das mensagens trocadas entre os familiares cuidadores e os profissionais de saúde foi realizada com auxílio do software Interface de R pour les Analyses Multidimensionnelle de Textes et de Questionnaires (IRAMUTEQ) utilizando a técnica da Classificação Hierárquica Descendente (CHD).

A seleção dos familiares cuidadores de pessoas com demência foi realizada no serviço de geriatria e gerontologia. Como critérios de inclusão foram selecionados os pacientes com acompanhamento regular e diagnóstico de demência ou doença de Alzheimer, além de diminuição da capacidade funcional. Não foram incluídos os pacientes com comprometimento cognitivo leve e funcionalidade preservada.

A amostra por conveniência foi composta 34 pessoas com demência e 34 familiares cuidadores acompanhados durante o período de 18 meses por meio do sistema. O monitoramento remoto das pessoas com demência foi realizado por cinco profissionais de saúde (três médicos residentes do serviço de geriatria, um enfermeiro e uma assistente social) responsáveis pela gestão do cuidado e suporte social aos cuidadores.

O Sistema é composto por dois aplicativos Android (SMAI Cuidador e SMAI Doutor), uma plataforma na internet (SMAI Web) e um servidor (SMAI Server). Foi desenvolvido pelo Laboratório de Ciência da Computação (LCC) da Universidade do Estado do Rio de Janeiro (UERJ) em parceria com o Núcleo de Atenção ao Idoso (NAI/HUPE/UERJ). O sistema encontra-se registrado no Instituto Nacional de Propriedade Industrial (INPI), como programa de computador (linguagem JAVA Android), sob o $\mathrm{n}^{\circ}$ BR 51.2015.000668-1. As figuras 1 e 2 apresentam a tela inicial do aplicativo SMAI Cuidador e o painel de funções, respectivamente. 


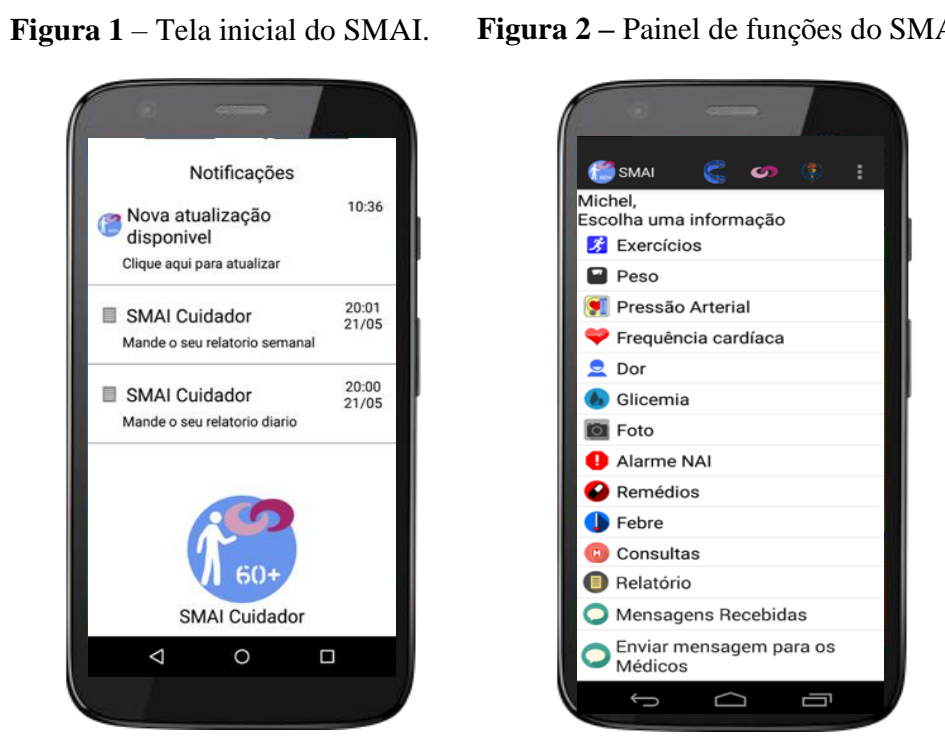

Fonte: Capturas de tela do aplicativo SMAI Cuidador.

Os dados foram coletados pelo aplicativo SMAI Cuidador, armazenados no servidor e organizados em uma planilha eletrônica. Foram incluídas na análise as mensagens trocadas no aplicativo SMAI entre os familiares cuidadores de pessoas com demência e os profissionais de saúde durante o período de utilização do aplicativo.

A análise ocorreu no período de abril a junho de 2020 com o auxílio do software IRAMUTEQ. Foram realizadas análises estatísticas sobre dois corpus textuais, sendo um deles resultado das mensagens enviadas pelos familiares cuidadores e outro com as respostas dos profissionais de saúde. Foi utilizada a versão 0.7 alpha 2, de livre licença, desenvolvida por Pierre Ratinaud em conjunto com o programa estatístico R, versão 3.5.1. O IRAMUTEQ permite cinco tipos de análise: estatísticas textuais clássicas; pesquisa de especificidades de grupo e análise fatorial; Classificação Hierárquica Descendente (CHD); análise de similitude de palavras e nuvem de palavras (Camargo \& Justo, 2013). Além disso, o software atua na construção de dendogramas, um tipo de diagrama que organiza fatores e variáveis a partir de um conjunto de textos.

Neste estudo, foi utilizada a técnica da CHD como estratégia para estimar a frequência das palavras por meio da classificação dos Segmentos de Textos (ST) em função dos vocabulários e análise lexical das palavras. O corpus foi organizado em um arquivo do Microsoft Word (.docx) e as categorias de palavras utilizadas na análise foram adjetivos, substantivos, verbos e outras formas. A partir dos ST atribuídos a cada uma das classes geradas pelo software, foi realizada a análise e interpretação dos dados para conhecer os temas relacionados ao cuidado de pessoas com demência a partir das mensagens trocadas entre os familiares cuidadores e os profissionais de saúde responsáveis pelo monitoramento remoto.

Foram obedecidos os critérios exigidos pela resolução no 466/2012 do Conselho Nacional de Saúde (CNS), obtendo aprovação de Comitê de Ética em Pesquisa (CEP) por meio do parecer no 2.013.410 e CAAE n 32654014.9.0000.5259. Os cuidadores que concordaram em participar do estudo assinaram o Termo de Consentimento Livre e Esclarecido (TCLE) e um termo de guarda do aparelho. Torna-se necessário esclarecer que não houve ônus para os participantes, uma vez que a aquisição dos smartphones e chips com plano de dados foi financiada com recursos do projeto SMAI com o apoio da Fundação de Amparo à Pesquisa do Estado do Rio de Janeiro (FAPERJ) e do CNPq (contratos E-26/110.243/2014 e 449861/2014-7, respectivamente). 


\section{Resultados}

O papel de cuidador foi desempenhado por familiares que moravam com os idosos e dividiam o cuidado com outras pessoas da família. Observou-se que os cuidadores tinham idade maior que 60 anos em média e a maioria era do sexo feminino. Com relação a escolaridade, houve predomínio de pessoas com mais de 12 anos de escolaridade (Tabela 1).

Tabela 1 - Características dos cuidadores participantes do estudo. Rio de Janeiro

(RJ), Brasil

\begin{tabular}{|c|c|}
\hline Características & \\
\hline Idade - média (DP) & $61,26( \pm 10,75)$ \\
\hline Sexo & $\mathrm{n}(\%)$ \\
\hline Feminino & $30(88)$ \\
\hline Masculino & $4(12)$ \\
\hline \multicolumn{2}{|l|}{ Estado Civil } \\
\hline Casado & $20(59)$ \\
\hline Viúvo & $3(9)$ \\
\hline Divorciado & $5(15)$ \\
\hline Solteiro & $6(17)$ \\
\hline \multicolumn{2}{|l|}{ Cor ou raça } \\
\hline Branca & $18(52)$ \\
\hline Preta & $8(24)$ \\
\hline Parda & $8(24)$ \\
\hline \multicolumn{2}{|l|}{ Anos de estudo } \\
\hline Até 8 & $5(15)$ \\
\hline 9 a 11 & $16(47)$ \\
\hline$>12$ & $13(38)$ \\
\hline \multicolumn{2}{|l|}{ Grau de Parentesco } \\
\hline Filho & $23(68)$ \\
\hline Cônjuge & $9(26)$ \\
\hline Irmão & $1(3)$ \\
\hline Nora & $1(3)$ \\
\hline \multicolumn{2}{|l|}{ Condição atual } \\
\hline Empregado & $4(12)$ \\
\hline Desempregado & $4(12)$ \\
\hline Autônomo & $1(3)$ \\
\hline Aposentado & $25(73)$ \\
\hline \multicolumn{2}{|c|}{ Renda Familiar (salários mínimos) } \\
\hline Até 2 & $10(29)$ \\
\hline 2 a 4 & $22(64)$ \\
\hline 4 a 6 & $2(7)$ \\
\hline \multicolumn{2}{|c|}{ Tempo como cuidador (anos) } \\
\hline Até 5 & $20(58)$ \\
\hline 6 a 7 & $5(15)$ \\
\hline 8 a 10 & $5(15)$ \\
\hline$>10$ & $4(12)$ \\
\hline \multicolumn{2}{|c|}{ Divide o cuidado do idoso } \\
\hline Sim & $21(62)$ \\
\hline Não & $13(38)$ \\
\hline \multicolumn{2}{|l|}{ Reside com o idoso } \\
\hline Sim & $29(85)$ \\
\hline Não & $5(15)$ \\
\hline
\end{tabular}

Fonte: Levantamento de dados. 
O grupo de participantes foi composto, na sua maioria, por filhos aposentados e com renda familiar entre 2 a 4 salários mínimos. A maioria dos familiares cuidadores se autodeclarou como cor ou raça branca, seguida de parda e preta. A Tabela 2 apresenta as características das pessoas com demência participantes do estudo.

Tabela 2 - Características das pessoas com demência participantes do estudo. Rio de Janeiro (RJ), Brasil.

\begin{tabular}{lr}
\hline Características & $\mathrm{n}(\%)$ \\
\hline Idade - média (DP) & $28(82)$ \\
\hline Sexo & $6(18)$ \\
\hline Feminino & \\
Masculino & $9(26)$ \\
\hline Estado Civil & $24(71)$ \\
\hline Casado & $1(3)$ \\
Viúvo & \\
Solteiro & $8(24)$ \\
\hline Escolaridade (anos) & $23(67)$ \\
\hline Analfabeto & $3(9)$ \\
\hline Até 8 anos & $6(17)$ \\
9 a 11 anos & $28(83)$ \\
\hline Incapacidade Funcional & \\
\hline Moderada & $14(42)$ \\
Severa & $20(58)$ \\
\hline Mobilidade Prejudicada & \\
\hline Sim & $27(79)$ \\
Não & $7(21)$ \\
\hline Diagnóstico Principal & $31(91)$ \\
\hline Doença de Alzheimer & $3(9)$ \\
\hline Outras demências & \\
\hline Sobrecarga do Cuidador & \\
\hline Sim & \\
Não & \\
\hline$\pm=$ Desvio Padrão & \\
\hline
\end{tabular}

Fonte: Levantamento de dados

Com relação aos idosos participantes do estudo, a idade média foi de 84 anos, com predomínio do sexo feminino, viúvos e diagnóstico de doença de Alzheimer e outras demências. Observa-se que a sobrecarga do cuidador está presente em $91 \%$ dos participantes e incapacidade funcional severa em $83 \%$, o que revela o grau de complexidade associado à tarefa de ser cuidador de uma pessoa com demência.

Após a análise do primeiro corpus textual no IRAMUTEQ foram identificados 34 textos, 24.579 ocorrências, 3.352 formas e 704 ST, dos quais 577 ST $(81,96 \%)$ foram aproveitados para a análise. Por meio da análise da CHD, foi criado um dendograma vertical com as palavras encontradas nos segmentos de textos organizados em cinco classes (Figura 3). Destas classes, emergiram cinco temas: organização das consultas e do cuidado; o cuidador monitorando a pessoa com demência; as rotinas de cuidado no domicílio; as alterações de comportamento e o cuidado com os medicamentos (Quadro 1). 
Figura 3 - Dendograma das mensagens dos familiares cuidadores no aplicativo SMAI Cuidador.

\begin{tabular}{|c|c|c|c|c|c|c|c|c|c|c|c|c|c|c|}
\hline \multicolumn{3}{|c|}{$\begin{array}{c}\text { Classe } 1 \\
149 / 577(25,82 \%)\end{array}$} & \multicolumn{3}{|c|}{$\begin{array}{c}\text { Classe 3 } \\
71 / 577(12,31 \%)\end{array}$} & \multicolumn{3}{|c|}{$\begin{array}{c}\text { Classe 2 } \\
127 / 577(22,01 \%) \\
\end{array}$} & \multicolumn{3}{|c|}{$\begin{array}{c}\text { Classe } 5 \\
115 / 577(19,93 \%)\end{array}$} & \multicolumn{3}{|c|}{$\begin{array}{c}\text { Classe } 4 \\
115 / 577(19,93 \%)\end{array}$} \\
\hline Palavra & $\mathbf{f}$ & $\mathbf{X}^{2}$ & Palavra & f & $\mathbf{X}^{2}$ & Palavra & f & $\mathbf{X}^{2}$ & Palavra & f & $\mathbf{X}^{2}$ & Palavra & f & $\mathbf{X}^{2}$ \\
\hline consulta & 49 & 76,6 & dormir & 32 & 67,46 & quando & 35 & 37,64 & bom & 83 & 40,95 & atenção & 24 & 50,02 \\
\hline médico & 26 & 54,34 & Tomar & 29 & 67,41 & momento & 16 & 29,09 & graça & 12 & 38,91 & bom & 83 & 40,95 \\
\hline ligar & 19 & 51,76 & hora & 21 & 61,94 & mão & 7 & 25,11 & deus & 16 & 31,8 & tosse & 17 & 32,87 \\
\hline levar & 36 & 49,85 & almoçar & 7 & 50,5 & pé & 12 & 24,08 & celular & 8 & 27,24 & noite & 62 & 32,68 \\
\hline exame & 26 & 48,38 & acordar & 18 & 48,39 & pressão & 15 & 21,97 & normal & 17 & 26,76 & apresentar & 23 & 31,22 \\
\hline hospital & 19 & 47,46 & café & 10 & 46,48 & fezes & 7 & 20,27 & noite & 57 & 21,13 & quadro & 10 & 27,07 \\
\hline próximo & 22 & 42,75 & deitar & 9 & 35,93 & gritar & 5 & 17,87 & paciente & 12 & 20,84 & então & 14 & 25,16 \\
\hline unati & 13 & 38,2 & agitado & 14 & 34,63 & passar & 25 & 17,59 & rivastigmina & 5 & 20,26 & menos & 14 & 25,16 \\
\hline mensagem & 25 & 35,97 & almoço & 9 & 32,47 & frente & 6 & 16,75 & errado & 5 & 20,26 & uso & 6 & 24,36 \\
\hline favor & 23 & 30,95 & manhã & 15 & 32,23 & pensar & 7 & 16,56 & problema & 8 & 19,59 & alteração & 12 & 23,0 \\
\hline marcar & 17 & 28,92 & leite & 4 & 28,71 & dersani & 4 & 14,27 & papai & 6 & 19,22 & comportamento & 13 & 21,98 \\
\hline resultado & 11 & 27,74 & troca & 4 & 28,71 & olhar & 4 & 14,27 & dever & 13 & 18,39 & \begin{tabular}{|l|} 
água \\
\end{tabular} & 12 & 20,84 \\
\hline data & 11 & 27,74 & alucinação & 4 & 28,71 & irritado & 4 & 14,27 & bem & 35 & 17,81 & começar & 20 & 20,57 \\
\hline gostar & 28 & 27,42 & recusar & 5 & 28,35 & sair & 8 & 14,24 & forte & 11 & 17,74 & piorar & 8 & 19,59 \\
\hline atender & 17 & 26,49 & cama & 11 & 28,01 & não & 85 & 14,22 & abraço & 9 & 17,69 & obrigar & 38 & 18,78 \\
\hline cirurgia & 9 & 26,26 & jantar & 9 & 24,47 & também & 18 & 14,2 & turvo & 4 & 16,18 & melhorar & 13 & 16,84 \\
\hline
\end{tabular}

Fonte: Tratamento dos dados no IRAMUTEQ, versão 0.7, alpha 2.

A classe 1, relacionada à organização das consultas e do cuidado apresentou predomínio em relação às demais (25,82\% dos ST), uma vez que foi observado que os cuidadores utilizavam as mensagens para tratar da agenda no ambulatório e gerenciar o retorno, o que revela a adesão dos usuários ao serviço. O sistema enviava alertas na forma de lembretes para os cuidadores próximo das consultas, sendo possível acompanhar a frequência nos atendimentos.

Quadro 1 - Síntese das mensagens dos cuidadores enviadas pelo aplicativo SMAI. Rio de Janeiro (RJ), Brasil.

\begin{tabular}{|c|c|c|c|}
\hline Classe & $\% \mathrm{ST}$ & Tema & Mensagens dos cuidadores no SMAI \\
\hline 1 & 25,82 & Organização das consultas e do cuidado & $\begin{array}{l}\text { Gostaria de saber se a consulta de rotina dela anteriormente agendada está } \\
\text { mantida a pergunta se faz para eu agendar os exames dela. (C04) } \\
\text { Gostaria de solicitar a consulta para final de setembro pois não fomos na } \\
\text { última porque ele estava resfriado e estava muito frio. (C15) }\end{array}$ \\
\hline 2 & 22,01 & $\begin{array}{l}\text { O cuidador monitorando a pessoa com } \\
\text { demência }\end{array}$ & $\begin{array}{l}\text { As coisas melhoraram bastante [...] a glicose também melhorou acho que foi } \\
\text { por conta da orientação que foi dada e está sendo respeitada. (C32) }\end{array}$ \\
\hline 3 & 12,31 & As rotinas de cuidado no domicílio & $\begin{array}{l}\text { Hoje, ela se engasgou no café, fiquei um pouco assustada. (CO2) } \\
\text { Ela desequilibrou e caiu na cozinha sem ferimentos aqui é o filho dela ela } \\
\text { teve uma queda no final da manhã e levei para o hospital, fez um curativo e } \\
\text { tomografia. (C05) }\end{array}$ \\
\hline 4 & 19,93 & As alterações de comportamento & $\begin{array}{l}\text { Hoje ele está muito alterado inquieto agressivo, já me machucou, não estou } \\
\text { mais aguentando. Não sei o que fazer, ele não tem mais condições de ir aí } \\
\text { está impossível levar ele. (C18) }\end{array}$ \\
\hline 5 & 19,93 & O cuidado com os medicamentos & $\begin{array}{l}\text { Paciente tem ficado prostrado gostaríamos de saber se poderemos suspender } \\
\text { o risperidona. }(\mathrm{C} 16)\end{array}$ \\
\hline
\end{tabular}

Fonte: Levantamento dos dados. 
Figura 4 - Dendograma das respostas dos profissionais aos familiares cuidadores no aplicativo SMAI.

\begin{tabular}{|c|c|c|c|c|c|c|c|c|c|c|c|c|c|c|}
\hline \multicolumn{3}{|c|}{$\begin{array}{c}\text { Classe } 4 \\
62 / 278(22,3 \%)\end{array}$} & \multicolumn{3}{|c|}{$\begin{array}{c}\text { Classe } 3 \\
79 / 278(28,42 \%) \\
\end{array}$} & \multicolumn{3}{|c|}{$\begin{array}{c}\text { Classe } 5 \\
53 / 278(19,06 \%) \\
\end{array}$} & \multicolumn{3}{|c|}{$\begin{array}{c}\text { Classe } 2 \\
44 / 278(15,83 \%)\end{array}$} & \multicolumn{3}{|c|}{$\begin{array}{c}\text { Classe 1 } \\
40 / 278(14,39 \%)\end{array}$} \\
\hline Palavra & f & \begin{tabular}{|l|}
$\mathbf{X}^{2}$ \\
\end{tabular} & Palavra & f & $\mathbf{X}^{2}$ & Palavra & f & \begin{tabular}{|l|}
$\mathbf{X}^{2}$ \\
\end{tabular} & Palavra & f & $\mathbf{X}^{2}$ & Palavra & f & $\mathbf{X}^{2}$ \\
\hline levar & 19 & 42,67 & dor & 15 & 28,53 & quadro & 8 & 29,39 & remarcar & 9 & \begin{tabular}{|l|}
49,47 \\
\end{tabular} & enviar & 15 & 52,59 \\
\hline emergência & 20 & 40,69 & problema & 17 & 23.23 & entrar & 10 & 26,2 & marcar & 16 & 37,62 & cirurgia & 8 & 49,01 \\
\hline cheiro & 8 & 23,8 & informação & 18 & 21,51 & alimento & 7 & 25,0 & agendar & 6 & 26,33 & foto & 13 & 38,76 \\
\hline família & 7 & 20,21 & obrigar & 24 & 21,45 & doença & 9 & 22,24 & dia & \begin{tabular}{|l|}
28 \\
\end{tabular} & 19,01 & instalar & 9 & 33,3 \\
\hline remédio & 6 & 16,66 & alimentar & 9 & 19,34 & sugerir & 5 & 21,62 & consulta & \begin{tabular}{|l|}
24 \\
\end{tabular} & 17,54 & agradecer & 9 & 26,78 \\
\hline realizar & 4 & 14,14 & bom & 65 & 18,87 & agitação & 5 & 21,62 & data & \begin{tabular}{|l|}
7 \\
\end{tabular} & 17,01 & atualização & 9 & 26,78 \\
\hline samu & 7 & 14,14 & motivo & 7 & 18,09 & social & 5 & 21,62 & confirmar & 9 & 16,87 & laudo & 4 & 24,15 \\
\hline lado & 4 & 14,14 & relatório & 20 & 16,24 & algo & 5 & 21,62 & agenda & 4 & 15,74 & envio & 5 & 23,66 \\
\hline ruim & 4 & 14,14 & febre & 10 & 13,41 & comprimido & 7 & 20,78 & bom & 39 & 15,51 & importância & 4 & 17,79 \\
\hline tomar & 7 & 13,62 & noite & 39 & 13,2 & evitar & 6 & 20,67 & manhã & 6 & 15,19 & aplicativo & 8 & 17,48 \\
\hline ajudar & 6 & 13,2 & notícia & 17 & 13,19 & contato & 11 & \begin{tabular}{|l|}
19,93 \\
\end{tabular} & uerj & 4 & 11,9 & smai & 9 & 16,39 \\
\hline informe & 6 & 13,2 & medicação & 10 & 11,4 & neurologista & 4 & 17,23 & receber & 7 & 11,32 & sistema & 4 & 13,61 \\
\hline forte & 5 & 13,18 & perto & 4 & 10,22 & próprio & 4 & 17,23 & hospital & 3 & 10,67 & pressão & 7 & 13,41 \\
\hline longo & 6 & 13,18 & não & 35 & 9,8 & cair & 4 & 17,23 & nai & 12 & 9,51 & favor & 12 & 12,87 \\
\hline avaliar & 12 & 12,91 & alarme & 5 & 9,09 & assistente & 4 & 17,23 & informar & 6 & 8,08 & pele & 3 & 12,1 \\
\hline chamar & 3 & 10,57 & melhorar & 11 & 8,71 & só & 5 & 16,42 & doutor & 6 & 5,99 & lesão & 3 & 12,1 \\
\hline
\end{tabular}

Fonte: Tratamento dos dados no IRAMUTEQ, versão 0.7, alpha 2.

O SMAI apresentava uma seção destinada ao registro da glicemia e da pressão arterial sistêmica, entretanto, os cuidadores optaram por informar os profissionais de saúde essas informações diretamente através das mensagens. Esses dados eram utilizados como base nas consultas para formular o plano terapêutico dos pacientes acompanhados pelo serviço. Com frequência, os familiares cuidadores utilizavam o sistema para o envio de mensagens aos profissionais a respeito das rotinas de cuidado no domicílio, sendo observados relatos de disfagia e queda. No que diz respeito à sobrecarga dos cuidadores, alguns deles utilizavam o recurso das mensagens como forma de desabafo e pedido de auxílio dos profissionais do serviço no manejo das alterações de comportamento e outros aspectos relacionados à organização do cuidado. Nesses casos, era realizado o acolhimento e encaminhamento desses cuidadores para os profissionais da Psicologia e Serviço Social.

As alterações de comportamento como agitação, agressividade e alucinações representaram um tema frequente no cuidado à pessoa com demência. Os familiares cuidadores enviaram mensagens com dúvidas a respeito dos medicamentos prescritos para o tratamento da demência e as drogas psicotrópicas utilizadas no controle das alterações comportamentais. Nos casos em que o familiar apresentava dificuldade de comparecimento ao serviço com a pessoa devido o estágio avançado da demência, o monitoramento remoto realizado por meio do aplicativo SMAI resultou no encaminhamento para o Programa de Atenção Domiciliar ao Idoso (PADI) para a continuidade da atenção.

O segundo corpus textual analisado com o auxílio do IRAMUTEQ foi constituído por 34 textos, 12.532 ocorrências, 1.297 formas e 359 ST, sendo aproveitado um total de 278 ST (77,44\%). A figura 4 apresenta as respostas dos profissionais organizadas em cinco classes. Delas, emergiram cinco temas associados às respostas sobre as consultas, sobrecarga do cuidador, organização do cuidado, manejo das alterações de comportamento e medicamentos, respectivamente.

A classe 3, que reuniu as respostas dos profissionais relacionadas à organização do cuidado, obteve maior frequência conforme apresentando no dendograma (28,42\% dos ST), o que demonstra uma necessidade dos cuidadores de pessoas com demência nesse tema. Os profissionais responderam os cuidadores a respeito das demandas fornecendo orientações sobre estratégias de manejo do paciente durante a alimentação e nos casos de risco de infecção urinária e desidratação, episódios de febre, dor e uso de medicamentos (Quadro 2). 
Quadro 2 - Síntese das respostas dos profissionais aos familiares cuidadores. Rio de Janeiro (RJ), Brasil.

\begin{tabular}{|c|c|c|c|}
\hline Classe & $\% \mathrm{ST}$ & Tema & Respostas dos profissionais no SMAI \\
\hline 1 & 14,39 & Respostas sobre as consultas & $\begin{array}{l}\text { Quando foi a última consulta e quando será a próxima? já tem algum dia } \\
\text { marcado? (RCO2) }\end{array}$ \\
\hline 2 & 15,83 & $\begin{array}{l}\text { Respostas sobre a sobrecarga do } \\
\text { cuidador }\end{array}$ & $\begin{array}{l}\text { Vi no seu relatório que tem se sentido estressada e cansada, nós, enquanto } \\
\text { equipe, somos sensíveis a essa situação e compreendemos que náo é fácil [...] } \\
\text { gostaria de ratificar a disponibilidade do serviço de psicologia como um espaço } \\
\text { de escuta sensível para suas demandas. (RC26) }\end{array}$ \\
\hline 3 & 28,42 & $\begin{array}{l}\text { Respostas sobre a organização do } \\
\text { cuidado }\end{array}$ & 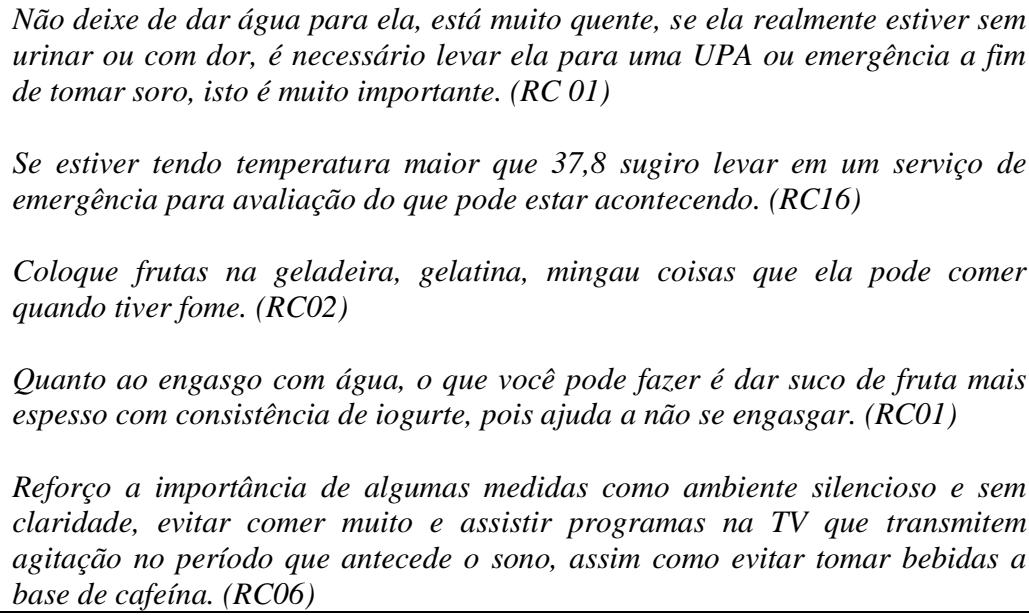 \\
\hline 4 & 22,3 & $\begin{array}{l}\text { Respostas sobre o manejo das } \\
\text { alterações de comportamento }\end{array}$ & $\begin{array}{l}\text { Evite entrar em conflito, estamos tentando contato com o neurologista para que } \\
\text { você possa retornar com ele ao ambulatório para ajustar a medicação. (RC18) } \\
\text { o que é recomendado fazer são mudanças no dia a dia mesmo além de muita } \\
\text { paciência e tranquilidade para lidar com isso, pois sabemos que é difícil. } \\
(R C 26)\end{array}$ \\
\hline 5 & 19,06 & $\begin{array}{l}\text { Respostas sobre o manejo dos } \\
\text { medicamentos }\end{array}$ & $\begin{array}{l}\text { Provavelmente não conseguiremos que ela fique calma todo o tempo, pois muito } \\
\text { do comportamento que ela apresenta é consequência da própria doença e } \\
\text { melhora pouco com os medicamentos. (RC26) } \\
\text { Não há medicação para tratar todas estas alterações. (RC18) } \\
\text { Temos um limite em relação a questão de medicamentos para resolver a questão } \\
\text { do banho e das refeiçôes, sabemos do seu esforço e dedicação, mas é preciso } \\
\text { compreender que existe a evolução da doença. (RC15) }\end{array}$ \\
\hline
\end{tabular}

Fonte: Levantamento dos dados.

Os profissionais do serviço aproveitaram o campo das mensagens para enviar orientações sobre a importância da vigilância nos casos de desidratação e risco de infecção que podem piorar o comportamento pessoa com demência. Nesses casos, eles eram orientados a procurar um pronto atendimento, uma vez que o sistema não atendia situações de urgência e emergência. Além disso, forneceram orientações sobre o uso de estratégias para melhorar a aceitação da alimentação e nos casos de disfagia e constipação. Essas informações são importantes no contexto do cuidar de uma pessoa com demência, pois preparam o cuidador para situações do cotidiano, onde a pessoa com demência pode apresentar distúrbios relacionados à alimentação e funcionamento intestinal.

Com relação aos relatos de sobrecarga do cuidador, foi verificada uma preocupação dos profissionais no sentido de oferecer suporte social e psicológico como forma de minimizar a sobrecarga. Uma das principais causas de estresse dos familiares cuidadores estava relacionada às alterações comportamentais dos pacientes sob seus cuidados. Sendo assim, as respostas dos profissionais focaram na orientação de evitar o conflito com a pessoa demência, buscando compreender a natureza dessas alterações que estão relacionadas à progressão da doença, além de utilizar-se de estratégias não farmacológicas para lidar com a situação. As respostas dos profissionais também apresentaram orientações sobre o manejo dos medicamentos para controle das alterações comportamentais e a progressão da demência ou doença de Alzheimer. 
Os profissionais utilizaram as mensagens para perguntar sobre os atendimentos no serviço e gerenciar o retorno para avaliação dos problemas relatados nas mensagens. A utilização do sistema facilitou a antecipação de consultas que aconteceriam em noventa dias ou mais, o que mostra a importância do monitoramento remoto e suporte aos cuidadores. Portanto, o cuidar de uma pessoa com demência se configura como uma atividade complexa e exaustiva que requer apoio social e assistencial aos familiares cuidadores.

\section{Discussão}

No presente estudo, foi possível conhecer as principais demandas de cuidados apresentadas pelos familiares cuidadores de pessoas com demência, sobretudo, aquelas relacionadas à organização do cuidado em si. A análise das mensagens dos cuidadores apontou para uma preocupação com os atendimentos no serviço, evidenciando um fator positivo relacionado a adesão terapêutica e uma demanda por suporte da rede de atenção à saúde. O modo como os familiares interagiram com os profissionais através das mensagens trocadas no ambiente digital fortaleceu o vínculo destes usuários com o serviço de saúde. O perfil dos participantes assemelha-se ao relato da literatura, onde a maioria dos cuidadores são pessoas do sexo feminino e familiares da pessoa idosa (Figueiredo, et al., 2018).

O suporte social tem o potencial de minimizar condições como a sobrecarga e o desgaste biopsicossocial dos cuidadores de pessoas idosas em situação de dependência funcional. As pessoas com demência tornam-se muito dependentes de seus cuidadores para acessar e utilizar os serviços de saúde. Por esta razão, os profissionais precisam apoiar os cuidadores no sentido de garantir suporte e orientação adequados (Hale, et al., 2020). Autores defendem a família como o principal recurso que o cuidador dispõe no processo do cuidado de pessoas com demência, mas existe uma demanda por suporte especializado e científico (Sena, et al., 2009). O envelhecimento com dependência impacta a dinâmica dos familiares que se tornam os responsáveis pelos cuidados da pessoa com demência. A situação de vulnerabilidade dessas pessoas idosas impõe às famílias rotinas de cuidados domiciliares complexas e invisíveis à sociedade, o que expõe a fragilidade do sistema de saúde e das políticas públicas na garantia de direitos sociais (Caldas, 2003; Neri \& Vieira, 2013).

No país, a prática da telemedicina está autorizada pela lei federal no 13.989/2020 (Brasil, 2020). A pandemia de Covid-19 provocou uma crise sanitária fazendo com que muitos serviços de saúde suspendessem suas atividades presenciais. com isso, observa-se um impacto significativo no tratamento de doenças crônicas como câncer, hipertensão, diabetes, Doença Pulmonar Obstrutiva Crônica (DPOC) e doenças renais. Essas pessoas apresentam são mais vulneráveis devido suas comorbidades e, por isso, têm maior probabilidade de desenvolver as formas mais graves da Covid-19 (Estrela, 2020).

No que diz respeito à população idosa, as alterações do envelhecimento na função imunológica e na resposta inflamatória aumentam a condição de vulnerabilidade ao novo Coronavírus. Condições de saúde como demência, acidente vascular cerebral e fraturas tornam os idosos mais expostos à infecção e embolia associadas à Covid-19 (Alves, Nunes \& Santos, 2021). Essa condição mundial exigiu novas abordagens e estratégias para a garantia de um cuidado adequado e seguro às pessoas com demência. Nesse sentido, dispositivos de telemedicina no campo da saúde móvel (mobile health), como smartphones, podem ser muito úteis no monitoramento e cuidados remotos, inclusive no acompanhamento domiciliar e suporte social a pacientes e familiares cuidadores. Essa revolução digital tem sido cada vez mais necessária, servindo como uma ferramenta de suporte social e assistencial no cuidado de pessoas com demência e seus cuidadores. Os aplicativos de saúde se destacam como um instrumento de monitoramento, informação e promoção de hábitos saudáveis podendo beneficiar familiares, cuidadores e profissionais (Amorim, et al., 2018).

Os familiares cuidadores foram orientados, desde o início, que o monitoramento clínico e remoto ofertado através do aplicativo SMAI não substituiria o atendimento presencial no serviço. Desta forma, o sistema foi considerado um plus para as pessoas com demência e seus cuidadores. As ações implementadas pelos profissionais do serviço de geriatria e gerontologia 
tiveram foco no suporte social dos cuidadores e no processo de gestão do cuidado na demência. Portanto, a dimensão da interprofissionalidade foi percebida como fundamental na produção do cuidado pela equipe de saúde, uma vez que os cuidadores se expressaram no aplicativo com demandas sociais e psicológicas que transformaram o modo de agir, as relações e o jeito de manejar os casos (Bernardo, 2016).

Outro tema observado foi a sobrecarga do cuidador que emergiu no escopo das mensagens e necessitou de atenção por parte de toda equipe do serviço. Com a progressão da demência, aumenta a demanda de cuidados e a necessidade de supervisão constante, papel desempenhado, muitas vezes, por um membro da família (Nascimento \& Figueiredo, 2019; Li, et al. 2020). Diante de demandas de cuidados complexas onde os familiares necessitam de orientação profissional e suporte social, foram observadas nas mensagens dos cuidadores relatos sobre mudanças na qualidade de vida, inclusive na vida pessoal e laboral. Sendo assim, o aumento do grau de dependência funcional impactou diretamente no comparecimento dos pacientes ao serviço e na condição de sobrecarga dos cuidadores. Observou-se que alguns familiares cuidadores apresentavam nível de sobrecarga moderado a severo e uma rede de suporte frágil sem ter com quem dividir o cuidado. Nesses casos, foi oferecido acompanhamento com a psicologia do serviço, enquanto a assistente social articulava o encaminhamento dos pacientes com demencia mais avançada para um programa de atendimento domiciliar, visando minimizar a sobrecarga desse cuidador.

As mensagens trocadas entre os familiares cuidadores e os profissionais foram utilizadas como base no plano terapêutico dos pacientes durante as consultas no serviço. Em alguns casos, o profissional antecipava o retorno de modo que eles pudessem ser reavaliados em menor tempo para a resolução de problemas identificados nas mensagens enviadas pelo aplicativo SMAI. Os profissionais de saúde, dentre eles, os enfermeiros exercem um papel importante na sociedade de promover a educação em saúde e orientar os familiares de pessoas com demência acerca dos cuidados domiciliares (Ramos, et al., 2015).

As respostas dos profissionais aos familiares cuidadores por meio do aplicativo concentraram-se na organização do cuidado da pessoa com demência no domić́lio, incluindo o manejo das alterações comportamentais, muito frequentes na demência, e dos medicamentos. Dentre essas alterações, destacam-se a agressividade, as alucinações e a perambulação, além da incontinência urinária e fecal que têm impacto significativo na sobrecarga e nas relações de cuidado (Santana, et al., 2018).

Foi observado na literatura que o uso de estratégias de saúde como o telecuidado para idosos com doença de Alzheimer e seus cuidadores têm o potencial de melhorar as habilidades de manejo das alterações de comportamento do idoso, além de contribuir para a percepção de sobrecarga do cuidador (Samus, et al., 2018). O telecuidado foi destacado por Santana et al. (2020) no acompanhamento pós-alta de pessoas com demência e seus cuidadores. Os resultados demonstraram uma diminuição da sobrecarga e do desgaste dos cuidadores, além da manutenção da capacidade funcional. O que reforça a importância das orientações às famílias e cuidadores, além da oferta de apoio social e assistencial dos profissionais e serviços de saúde especializados em saúde do idoso. O aplicativo SMAI contribuiu para o acompanhamento dessas demandas de saúde, sendo possível gerenciar cuidados relacionados à disfagia, alimentação, constipação, desidratação e estratégias nãofarmacológicas de manejo das alterações de comportamento.

\section{Considerações Finais}

$\mathrm{O}$ sistema funcionou como uma ferramenta inovadora de suporte social e assistencial aos familiares cuidadores que utilizaram a troca de mensagens com a equipe de saúde, recebendo orientações acerca do cuidar de uma pessoa com demência e suas especificidades. Esse apoio oferecido pelo aplicativo se mostrou como um exemplo de tecnologia móvel que estimula o acompanhamento domiciliar e contribui para o processo de gestão e organização do cuidado.

A utilização do aplicativo SMAI possibilitou o suporte social dos familiares cuidadores que se traduziu na organização das consultas; no manejo das alterações comportamentais; no monitoramento remoto e no gerenciamento do 
cuidado pela equipe de saúde, além do estreitamento do vínculo e melhoria da comunicação, apresentando um impacto positivo na produção do cuidado do serviço de geriatria e gerontologia.

O acompanhamento domiciliar através do aplicativo SMAI resultou em um modelo híbrido de atendimento (presencial e remoto) que se configurou como uma prática transformadora para a gestão do cuidado em saúde. Esse modelo de atenção à saúde tem se destacado no contexto da pandemia de Covid-19, no entanto, é importante ressaltar que essas tecnologias devem valorizar as realidades individuais e culturais das pessoas com demência e seus cuidadores, considerando suas necessidades sociais e de saúde.

A implantação do sistema no processo de trabalho do serviço de geriatria e gerontologia impactou positivamente na produção de um cuidado voltado às necessidades das pessoas com demência e suporte social dos cuidadores. O serviço possui programas de residências médica e multiprofissional em saúde do idoso com foco na integralidade e interprofissionalidade. A coordenação realizava, semanalmente, reuniões de equipe para o planejamento terapêutico das pessoas com demência acompanhadas pelo aplicativo SMAI, o que contribuiu para a melhoria na comunicação e a oferta de suporte na organização dos cuidados da pessoa com demência.

O uso de aplicativos móveis em saúde pode funcionar como uma ferramenta de suporte aos cuidadores de idosos, além de estimular o cuidado domiciliar e o convívio na comunidade com as famílias e amigos, prevenindo a institucionalização precoce e o isolamento social. A reorientação do modelo de atenção atual surge como uma das estratégias globais do cuidado centrado na pessoa com demência, onde são valorizadas as capacidades emocionais e cognitivas remanescentes. Para isso, é necessário integração dos profissionais de saúde, familiares e cuidadores envolvidos no processo de cuidar para que a pessoa com demência seja vista em seu contexto familiar, cultural, étnico e de gênero.

Nesse sentido, as transformações tecnológicas e sociais podem contribuir para o surgimento de modelos de cuidados à saúde cada vez mais inovadores. A prática da saúde móvel, caracterizada pelo uso de aplicativos de saúde e sistemas de telecuidado, se configura como uma atividade em expansão nesse contexto epidemiológico, entretanto, ainda são incipientes os estudos de validação e avaliação do impacto na saúde pública, visando a promoção da saúde e a prevenção da hospitalização e institucionalização precoces. Portanto, o estudo abre perspectivas para o desenvolvimento de aplicativos móveis que promovam o cuidado integral das pessoas com demência e ofereçam uma rede de suporte social aos familiares cuidadores.

\section{Referências}

ADI. (2015). Alzheimer's Disease International. <https://www.alzint.org/resource/world-alzheimer-report-2015/>

Alves, T. O., Nunes, W. A. S. \& Santos, M. V. F. (2021). Impacto da pandemia do Covid-19 na saúde dos idosos e intervenção da equipe de enfermagem. Research, Society and Development, 10 (14), 1-9.

Amorim, D. N. P. et. al. (2018). Aplicativos móveis para a saúde e o cuidado de idosos. Rev Eletron Comun Inf Inov Saúde, 12 (1), 58 -71.

Bernardo, M. H. J. (2016). Envelhecimento com dependência: a invisibilidade dos cuidados familiares. Cuidado e Interprofissionalidade: uma experiência de atenção integral à saúde da pessoa idosa, $\mathrm{CRV}$.

Brasil. (2020). Lei $\mathrm{n}^{\circ}$ 13.989, de 15 de abril de 2020. Dispõe sobre o uso da telemedicina durante a crise causada pelo Coronavírus (SARS-cov-2). <https://www.in.gov.br/en/web/dou/-/lei-n-13.989-de-15-de-abril-de-2020-252726328>

Caldas, C. P. (2003). Envelhecimento com dependência: responsabilidades e demandas da família. Cad. Saúde Pública, 19 (3), $773-781$.

Camargo, B. V. \& Justo, A. M. (2013). IRAMUTEQ: Um Software Gratuito para Análise de Dados Textuais. Temas em Psi, 1 (2), $513-518$.

Cuffaro, L. et. al. (2020). Dementia care and COVID-19 pandemic: a necessary digital revolution. Neurol Sci, 41 (8), $1977-1979$.

Carnut, L. (2017). Cuidado, integralidade e atenção primária: articulação essencial para refletir sobre o setor saúde no Brasil. Saúde debate, 41 (115), 11771186.

Estrela, F. M. et. al. (2020). COVID-19 e doenças crônicas: impactos e desdobramentos frente à pandemia. Rev baiana enferm, 34 (1), 1 -7.

Figueiredo, K. et. al. (2018). Efeito de intervenção de exercícios físicos multifuncionais na percepção da qualidade de vida de cuidadores de pacientes com Doença de Alzheimer. Rev Bras Qual Vida, 19 (3), 29-49. 
Research, Society and Development, v. 11, n. 2, e52311226073, 2022

(CC BY 4.0) | ISSN 2525-3409 | DOI: http://dx.doi.org/10.33448/rsd-v11i2.26073

Hale, L. et. al. (2020). Constructing Normalcy in Dementia Care: Carers' Perceptions of Their Roles and the Supports They Need. The Gerontologist, 60 (5), 905-915.

IBGE. (2018). Instituto Brasileiro de Geografia e Estatística. Projeções da população: Brasil e unidades da federação: revisão 2018. $<$ https://biblioteca.ibge.gov.br/index.php/biblioteca-catalogo?view=detalhes\&id=2101597>

Li, Y. et. al. (2020). Health literacy, social support, and care ability for caregivers of dementia patients: structural equation modeling. Geriatr Nurs, 41 (5), 600-607.

Menezes, E. L. C. et. al. (2020). Modos de produção do cuidado e a universalidade do acesso - análise de orientações federais para o trabalho das equipes da APS no brasil. Ciênc saúde coletiva, 25 (5), 1751-1763.

Mueller, A. L. McNamara, M. S. \& Sinclair, D. A. (2020). Why does COVID-19 disproportionately affect older people? Aging, 12 (10), $9959-9981$.

Nascimento, H. G. \& Figueiredo, A. E. B. (2019). Demência, familiares cuidadores e serviços de saúde: o cuidado de si e do outro. Ciênc saúde colet, 24 (4), $1381-1392$.

Neri, A. L. \& Vieira, L. A. M. (2013). Envolvimento social e suporte social percebido na velhice. Rev Bras Geriatr Gerontol, 16 (3), 419-432.

Ohannessian, R. Duong, T. A. \& Odone, A. (2020). Global telemedicine implementation and integration within health systems to fight the COVID-19 pandemic: a call to action. JMIR Public Health Surveill, 6 (2), 1-4.

Polit, D. F. \& Beck, C. T. (2011). Fundamentos de Pesquisa em Enfermagem: avaliação de evidências para a prática de enfermagem. Porto Alegre: Artmed.

Porter, E. J. (2007). Problems with preparing food reported by frail older women living alone at home. Adv Nurs Sci, 30 (2), 159-74

Ramos, A. K. et. al. (2015). Gerenciamento do cuidado de enfermagem ao idoso com Alzheimer. Rev Cubana Enfermer, 31 (4), 1-12.

Rathnayake, S. et. al. (2019). Family carers' perspectives of managing activities of daily living and use of mHealth applications in dementia care: A qualitative study. J Clin Nurs, 28 (23-24), 4460-4470.

Rathnayake, S. et. al. (2019). mHealth applications as an educational and supportive resource for family carers of people with dementia: An integrative review. Dementia, 18 (1), 3091-3112.

Samus, Q. M. et. al. (2018). Home is where the future is: The BrightFocus Foundation consensus panel on dementia care. Alzheimers Dement, 14(1), 104-114.

Santana, R. F. et. al. (2018). Telecuidado para idosos com Alzheimer e seus cuidadores: revisão sistemática. cienc cuid saúde, 17 (4), 1-6.

Santana, R. F. et. al. (2020). Telecuidado no acompanhamento pós-alta de idosos com demência e seus cuidadores: quase-experimental. Online braz j nurs, 19 (2), 1-22.

Sena, E. L. S. et. al. (2009). Caracterização e contribuições do suporte social aos cuidadores de pessoas com doença de Alzheimer. Memorialidades, 12 (1), $129-150$.

Silva, R. H. et. al. (2020). Aplicativos de saúde para dispositivos móveis: uma revisão integrativa. Braz J H Rev, 3 (5), 11754-11765.

Sim, I. (2019). Mobile devices and health. N Engl J Med, 381 (10), 956-968.

Yousaf, K. et. al. (2019). Mobile-Health Applications for the Efficient Delivery of Health Care Facility to People with Dementia (PwD) and Support to Their Carers: A Survey. BioMed R Int, (1), 1-26. 